\title{
HYPERBOLIC GEOMETRY: THE FIRST 150 YEARS
}

\author{
BY JOHN MILNOR
}

This will be a description of a few highlights in the early history of non-euclidean geometry, and a few miscellaneous recent developments. An Appendix describes some explicit formulas concerning volume in hyperbolic 3-space.

The mathematical literature on non-euclidean geometry begins in 1829 with publications by N. Lobachevsky in an obscure Russian journal. The infant subject grew very rapidly. Lobachevsky was a fanatically hard worker, who progressed quickly from student to professor to rector at his university of Kazan, on the Volga.

Already in 1829, Lobachevsky showed that there is a natural unit of distance in non-euclidean geometry, which can be characterized as follows. In the right triangle of Figure 1 with fixed edge $a$, as the opposite vertex $A$ moves infinitely far away, the angle $\theta$ will increase to a limit $\theta_{0}$ which is assumed to be strictly less than $\pi / 2$. He showed that

$$
a=-\log \tan \left(\theta_{0} / 2\right)
$$

if the unit of distance is suitably chosen. In particular,

$$
a \approx(\pi / 2)-\theta_{0}
$$

if $a$ is very small. (In the interpretation introduced by Beltrami forty years later, this unit of distance is chosen so that curvature $\equiv-1$.)

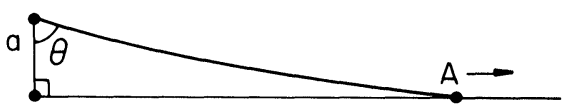

FiguRE 1. A right triangle in hyperbolic space

By early 1830, Lobachevsky was testing his "imaginary geometry" as a possible model for the real world. If the universe is non-euclidean in Lobachevsky's sense, then he showed that our solar system must be extremely small, in terms of this natural unit of distance. More precisely, taking the vertex $A$ in Figure 1 to be the star Sirius and taking the edge $a$ to be a suitably chosen radius of the Earth's orbit, he used the (unfortunately incorrect) estimate

$$
\pi-2 \theta \cong 1.24 \text { seconds of } \operatorname{arc} \cong 6 \times 10^{-6} \text { radians }
$$

Presented to the Symposium on the Mathematical Heritage of Henri Poincaré, April 7-10, 1980; received by the editors March 10, 1981 and, in revised form, April 20, 1981.

1980 Mathematics Subject Classification. Primary 01A55, 01A60, 51M10; Secondary 57R15, $20 \mathrm{H} 10$. 
for the parallax of Sirius to conclude that the diameter $2 a$ of Earth's orbit is less than $6 \times 10^{-6}$. (The correct parallax of $0.37^{\prime \prime}$ for Sirius would have given a sharper estimate. In fact, the first reliable measurements of parallax were made by Bessel several years later, in 1838.)

By late 1830, he was working out the volumes of pyramids, and other polyhedra in 3-dimensional non-euclidean space. Such computations are not easy, and can be quite interesting. (Compare the Appendix.)

A few years later, in 1832, J. Bolyai, a flamboyant officer in the AustroHungarian army, published an independent account of non-euclidean geometry. However, perhaps because he was discouraged by Gauss, he did not pursue the subject as far as Lobachevsky.

Although Lobachevsky and Bolyai were the first with the courage to publish, it should be noted that Gauss himself had been privately working on similar ideas for many years. In a letter to Taurinus in 1824 he wrote (roughly translated): ${ }^{1}$

"The assumption that the sum of the three angles [of a triangle] is smaller than $180^{\circ}$ leads to a geometry which is quite different from our (euclidean) geometry, but which is in itself completely consistent. I have satisfactorily constructed this geometry for myself so that I can solve every problem, except for the determination of one constant, which cannot be ascertained a priori. The larger one chooses this constant, the closer one approximates euclidean geometry. ... If non-euclidean geometry were the true geometry, and if this constant were comparable to distances which we can measure on earth or in the heavens, then it could be determined a posteriori. Hence I have sometimes in jest expressed the wish that euclidean geometry is not true. For then we would have an absolute a priori unit of measurement."

His words to Bessel, in 1830, have an even more modern ring:

" . . . we must admit with humility that, while number is purely a product of our mind, space has a reality outside of our mind, so that we cannot completely prescribe its laws a priori."

For the first forty years or so of its history, the field of non-euclidean geometry existed in a kind of limbo, divorced from the rest of mathematics, and without any firm foundation. However, Gauss' theory of curved surfaces [1827], and Riemann's theory of higher dimensional curved manifolds [1868] provided a way of integrating non-euclidean geometry into more respectable branches of mathematics. In fact Riemann briefly described the theory of manifolds of constant curvature $\alpha$, citing the metric

$$
d s=\sqrt{d x_{1}^{2}+\cdots+d x_{n}^{2}} /\left(1+\frac{\alpha}{4}\left(x_{1}^{2}+\cdots+x_{n}^{2}\right)\right)
$$

as an example.

The turning point came in 1868 , with the publication of two papers by $E$. Beltrami. In the first, Beltrami showed that two-dimensional non-euclidean geometry is nothing more nor less than the study of suitable surfaces of

\footnotetext{
${ }^{1}$ Gauss [1900, pp. 187, 201]. For other early workers in non-euclidean geometry, see for example Coxeter [1942].
} 
constant negative curvature. $\mathrm{H}_{\mathrm{S}}$ introduced the term pseudosphere of radius $R$ for a surface of curvature $-1 / R^{2}$. (In practice, he used the term "pseudosphere" only for complete, simply connected surfaces.) In this first paper, he was baffled by the 3-dimensional case. However, after encountering Riemann's inaugural address, which had been delivered in 1854 but published only after his death, in 1868, Beltrami published a second paper, on $n$-dimensional pseudospherical geometry. He started with what I will call the hemisphere model. Points of the $n$-dimensional non-euclidean geometry are identified with interior points of the hemisphere

$$
y=\sqrt{a^{2}-x_{1}^{2}-\cdots-x_{n}^{2}}, \quad y \geqslant 0
$$

in $(n+1)$-dimensional euclidean space, provided with the Riemannian metric

$$
d s=R \sqrt{d x_{1}^{2}+\cdots+d x_{n}^{2}+d y^{2}} / y .
$$

He noted that this model is simply connected, and that the "limit space", consisting of boundary points with $y=0$, is infinitely far from the interior in this metric. We will refer to such boundary points as points at infinity.

Projecting orthogonally down to the disk

$$
x_{1}^{2}+\cdots+x_{n}^{2} \leqslant a^{2},
$$

he showed that each pseudospherical geodesic maps precisely to a euclidean straight line segment. Thus he obtained the projective disk model, later popularized by Klein.

On the other hand, projecting the hemisphere stereographically onto a disk, he obtained the conformal disk model, with the metric

$$
d s=\sqrt{d \xi_{1}^{2}+\cdots+d \xi_{n}^{2}} /\left(1-\left(\xi_{1}^{2}+\cdots+\xi_{n}^{2}\right) / 4 R^{2}\right)
$$

which had been noted already by Riemann.

Finally, performing an inversion in a boundary point of this disk, he obtained the half-space model, with coordinates $x_{1}, \ldots, x_{n-1}$ and $y \geqslant 0$, and with metric $R\left(d x_{1}^{2}+\cdots+d x_{n-1}^{2}+d y^{2}\right)^{1 / 2} / y$. He pointed out that this half-space metric had been used already by Liouville in the 2-dimensional case.

Klein [1871] reinterpreted Beltrami's projective disk model in terms of projective geometry. Following Cayley [1859], he took as his starting point the expression

$$
\frac{1}{2} \log \frac{|q-a||b-p|}{|p-a||b-q|}
$$

for the non-euclidean distance between two points $p, q$, as illustrated in Figure 2. (The factor $1 / 2$ is inserted so that curvature will be -1 .) Here $|q-a|$ denotes the euclidean distance from $a$ to $q$. In this paper he introduced the term hyperbolic geometry for the non-euclidean geometry of Lobachevsky and Bolyai. 


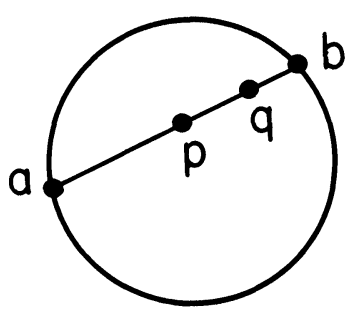

FIGURE 2. The Cayley formula for noneuclidean distance, in the projective disk model

Poincaré [1882] re-introduced the Liouville-Beltrami upper half-plane model; and used it to identify the group of orientation preserving isometries of the hyperbolic plane with the group, now usually called $P S L_{2} \mathbf{R}$, consisting of all fractional linear transformations

$$
z \mapsto(a z+b) /(c z+d)
$$

with real coefficients and with determinant +1 . As a foundation for the study of automorphic functions, he emphasized the importance of finding discrete groups of hyperbolic isometries. He noted (\$XIII) that examples of such discrete groups had been described already by Schwarz [1873], and others.

In [1883], Poincare showed that the analogous group of orientation preserving isometries of 3-dimensional hyperbolic space can be identified with the group $P S L_{2} \mathbf{C}$ consisting of all fractional linear transformations of the plane of points at infinity, with complex coefficients. Again he studied the problem of finding discrete subgroups.

Picard [1884] described one particularly interesting example. The ring $\mathbf{Z}[i] \subset \mathbf{C}$ of Gaussian integers gives rise to a discrete group $P S L_{2} \mathbf{Z}[i] \subset$ $P S L_{2}$ C, consisting of fractional linear transformations with Gaussian integer coefficients. A fundamental domain for this group, acting on hyperbolic 3-space, is noncompact, but has finite volume.

Bianchi [1891] described the analogous construction using the integers of an arbitrary imaginary quadratic number field. The precise volume of a corresponding fundamental domain was computed much later by Humbert [1919], in infinitely many cases. (See the Appendix.)

Inspired by examples due to Clifford, Klein [1890] posed the following problem:

"... to classify all connectivities which can possibly arise among closed manifolds of constant curvature."

It must be noted in this context that the concept of a (global) manifold was never defined in any satisfactory way during the nineteenth century. For smooth manifolds in euclidean space, the definition given by Betti [1871] works only if the normal bundle is trivial. Poincare [1895] used a similar definition and also suggested extending a coordinate patch by analytic continuation. Hadamard [1898] clarified the subject by giving a lucid description of surfaces in 3-space in terms of overlapping coordinate patches. Weyl 
[1913] paved the way for our modern concept of smooth manifold by defining an abstract surface in terms of overlapping coordinate patches. In particular, he gave a clear discussion of the universal covering surface. The generalization to $n$ dimensions was now straightforward. (For a particularly nice presentation, see Cartan [1928].) During the same span of time, the study of combinatorial manifolds progressed from the rather vague definitions of Dyck [1890] and Poincaré to the precise definition of Brouwer [1912]. (See also Tietze [1908], Veblen and Alexander [1913].) The related concept of topological space is due particularly to Hausdorff [1914].

Let us return to the study of manifolds of constant curvature. Killing [1891] related Klein's classification problem to the study of discrete groups of isometries, as follows. If $E$ is either $n$-dimensional hyperbolic space, $n$-dimensional euclidean space, or the $n$-dimensional sphere, and if $\Gamma$ is a discrete group of isometries which acts freely on $E$, then he showed that the quotient $E / \Gamma$ is a manifold of constant curvature. He called such a quotient a Clifford-Klein space form.

Much later, Hopf [1925] clarified this work by introducing a definition of completeness, showing that Killing's Clifford-Klein space forms are precisely the complete Riemannian manifolds of constant curvature. The concept of completeness was further developed by Cartan [1928] and Hopf-Rinow [1931].

By definition, a hyperbolic manifold is a Clifford-Klein manifold with curvature equal to -1 . In the years following Killing's paper, a number of examples of hyperbolic manifolds were described. Compare Borel [1963]. The study of such manifolds is currently an extremely active area of research, particularly due to the work of Thurston [1978, 1981].

Here let me describe two particularly simple examples. The first is due to Gieseking [1912]. Start with a regular hyperbolic 3-simplex $\Delta$ having all four vertices on the sphere of points at infinity. Each of the six dihedral angles of $\Delta$ is then equal to $\pi / 3$. (Compare the Appendix.) Now identify two faces of $\Delta$ by means of a rotation of $2 \pi / 3$ about a common vertex. Similarly, identify the opposite two faces by rotating about a common vertex. Then all six edges will be identified. Since the six dihedral angles add up to $2 \pi$, they fit smoothly together so that we obtain a non-singular hyperbolic manifold, even in a neighborhood of the common edge. This Gieseking manifold is nonorientable. It is noncompact, but is complete with finite volume.

The second example is due to Riley [1975]. Consider the figure eight knot $K$, as shown in Figure 3. The fundamental group $\Pi$ of the complement $S^{3}-K$ is generated by two loops $\alpha, \beta$, as illustrated, which are subject to a single defining relation $\left(\alpha \beta^{-1} \alpha^{-1} \beta\right) \alpha=\beta\left(\alpha \beta^{-1} \alpha^{-1} \beta\right)$. Suppose that we look for a faithful representation

$$
\Pi \rightarrow P S L_{2} \mathbf{C}
$$

and require that the image must be a discrete subgroup. The generators $\alpha$, $\beta \in \Pi$ will correspond to unknown matrices $A, B$, which must satisfy a single matrix equation. 


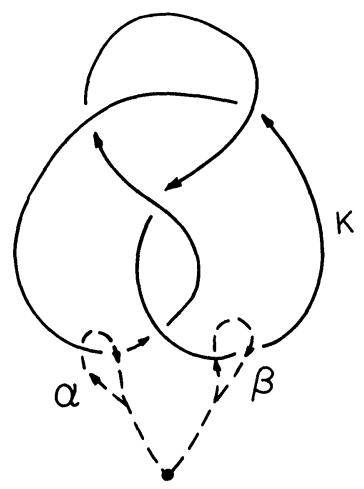

Figure 3. The figure eight knot

The possible choices for $A$ and $B$ can be drastically narrowed as follows. Note that every nontrivial element of $P S L_{2} \mathrm{C}$ is contained in a unique maximal abelian subgroup which is conjugate either to the group consisting of all diagonal matrices $\left[\begin{array}{ll}\lambda_{0} & 0 \\ 0 & \lambda^{-1}\end{array}\right]$ or to the group of all unipotent matrices of the form $\left[\begin{array}{ll}1 & \lambda \\ 0 & 1\end{array}\right]$, according as the given element does or does not have two linearly independent eigenvectors. Now the generator $\alpha$ of $\Pi$ is contained in a free abelian group $\mathbf{Z} \oplus \mathbf{Z} \subset \Pi$, corresponding to the fundamental group of the boundary of a tubular neighborhood of $K$. It is easy to check that $\mathbf{Z} \oplus \mathbf{Z}$ cannot be embedded as a discrete subgroup of the group of diagonal matrices, which is isomorphic to the multiplicative group $\mathbf{C}^{*}$. Hence $\alpha$, and similarly $\beta$, must map to unipotent elements of $P S L_{2} \mathbf{C}$, with eigenvalue 1 . Using a basis for $\mathbf{C}^{2}$ consisting of an eigenvector for $\alpha$ and an eigenvector for $\beta$, we may assume that

$$
\alpha \mapsto A=\left[\begin{array}{ll}
1 & 1 \\
0 & 1
\end{array}\right], \quad \beta \mapsto B=\left[\begin{array}{rr}
1 & 0 \\
-\omega & 1
\end{array}\right]
$$

where $\omega$ is some unknown complex constant. Now computation shows that these two matrices satisfy the required relation if and only if $1+\omega+\omega^{2}=0$, so that $\omega=(-1 \pm \sqrt{-3}) / 2$. Thus the requirement that the "peripheral subgroup" $\mathbf{Z} \oplus \mathbf{Z} \subset \Pi$ maps isomorphically to a discrete subgroup of $P S L_{2} \mathbf{C}$ leads to a homomorphism

$$
h: \Pi \rightarrow P S L_{2} \mathbf{C}
$$

which is uniquely determined, up to inner automorphism and complex conjugation.

Since the image $h(\Pi)$ is contained in the Bianchi group $P S L_{2} Z[\omega]$, it is certainly discrete in $P S L_{2}$ C. In fact Riley proves that $h$ maps $\Pi$ isomorphically onto a subgroup $h(\Pi) \subset P S L_{2} Z[\omega]$ of index twelve. Using the HakenWaldhausen theory of 3-manifolds, he then proves that the complement $S^{3}-K$ is actually homeomorphic to the hyperbolic manifold $H^{3} / h(\Pi)$.

In an interesting complement to these two examples, Thurston [1978] shows by direct geometric construction that $S^{3}-K$ is homeomorphic to the 2-fold orientable covering of the Gieseking manifold. In particular, the hyperbolic 
manifold $S^{3}-K$ has a kind of triangulation into two regular ideal hyperbolic 3-simplexes, with their boundaries pasted together by suitable isometries.

The uniqueness of this Riley representation $\Pi \rightarrow P S L_{2} \mathbf{C}$ illustrates the following fundamental result.

RIGIDITY THEOREM. If two hyperbolic manifolds of finite volume, with dimension $n \geqslant 3$, have isomorphic fundamental groups, then they must necessarily be isometric to each other.

In the compact case, this was proved by Mostow [1971, 1973]. (See also Margulis [1970].) For the noncompact case, it was proved by Prasad [1973], making essential use of Mostow's work.

It follows that geometric invariants such as volume, the lengths of closed geodesics, and the eigenvalues of the Laplacian operator, are also topological invariants. For an example to show that these particular geometric invariants are not sufficient to determine the topology of a hyperbolic 3-manifold, see Vignéras [1980].

In contrast to this Mostow-Prasad rigidity theorem, Thurston has proved a fundamental result which can be stated in a preliminary form as follows.

NoNRIGIDITY THEOREM. Suppose that $M=H^{3} / \Gamma$ is an orientable hyperbolic 3-manifold which is noncompact, but has finite volume. Then there exists an infinite sequence of hyperbolic manifolds

$$
M_{j}=H^{3} / h_{j}(\Gamma)
$$

which have strictly smaller volume, and which approximate the original manifold $M$ as $j \rightarrow \infty$ in the sense that the homomorphisms

$$
h_{j}: \Gamma \rightarrow P S L_{2} \mathbf{C}
$$

tend to the inclusion $\Gamma \subset P S L_{2} \mathrm{C}$ in the topology of pointwise convergence. Furthermore, the volumes of the $M_{j}$ tend to the volume of $M$.

Of course, according to the Rigidity Theorem, these approximating manifolds $M_{j}$ cannot be homeomorphic to $M$, or to each other. In particular, the representations $h_{j}$ cannot be faithful. Their behavior can be described more precisely as follows.

To fix our ideas, suppose that the noncompact manifold $M$ has just one end. Then a neighborhood of infinity in $M$ is smoothly covered by a unique field of half-infinite geodesics, which converge exponentially towards each other as we go out to infinity. Any orthogonal trajectory to this field of geodesics is a flat torus. The fundamental group of such a torus-near-infinity is called a peripheral subgroup $\mathbf{Z} \oplus \mathbf{Z}$, embedded in the fundamental group $\Gamma$ of $M$.

As in the discussion of Riley's example, the original representation $\Gamma \subset$ $P S L_{2} \mathbf{C}$ must map this peripheral subgroup $\mathbf{Z} \oplus \mathbf{Z}$ to a unipotent subgroup, having just one eigenvector with a double eigenvalue of 1 . If we perturb this representation slightly within the space of all homomorphisms $\Gamma \rightarrow P S L_{2} \mathrm{C}$, then the single eigenvector will split into two nearby but linearly independent eigenvectors. Hence the subgroup $\mathbf{Z} \oplus \mathbf{Z}$ will map into some subgroup of 
$\mathrm{PSL}_{2} \mathrm{C}$ which is isomorphic to the multiplicative group $\mathrm{C}^{*}$. Such a representation of $\mathbf{Z} \oplus \mathbf{Z}$ cannot be faithful, with discretely embedded image. For most choices of perturbation, the image of $\mathbf{Z} \oplus \mathbf{Z}$ will simply be a nondiscrete subgroup of $\mathbf{C}^{*}$. But for certain carefully chosen perturbations $h=h_{p q}$, the images of the two generators $x, y$ of $\mathbf{Z} \oplus \mathbf{Z}$ will be subject to a new relation of the form $h\left(x^{p} y^{q}\right)=I$; and these images will generate a discretely embedded free cyclic subgroup of $\mathbf{C}^{*}$. In fact Thurston shows that such a perturbation exists whenever $p, q$ are relatively prime integers with $|p|+|q|$ sufficiently large. Furthermore, the image $h_{p q}(\Gamma)$ is always a discrete subgroup of $P S L_{2} \mathrm{C}$; and $h_{p q}$ tends to the inclusion homomorphism as $|p|+|q| \rightarrow \infty$.

Topologically, the resulting quotient manifold $M_{p q}=H^{3} / h_{p q}(\Gamma)$ can be obtained from $M$ by cutting off a neighborhood of infinity bounded by a flat torus $S^{1} \times S^{1}$ and pasting in its place a solid torus $S^{1} \times D^{2}$ which is attached in such a way as to introduce the required relation $x^{p} y^{q}=1$ into the fundamental group. Thurston calls this operation a Dehn surgery.

Thus, if $M$ has only one end, the approximating manifolds $M_{p q}$ will always be compact. If $M$ has several ends, then one or more of them can be capped off by analogous Dehn surgeries. In particular, $M$ can always be approximated arbitrarily closely (in a suitable sense) by compact hyperbolic manifolds.

Here is a concluding example to test the reader's powers of visualization. Wielenberg [1978] has shown that the complement of the Whitehead link $W$ of Figure 4 can be given the structure of a hyperbolic manifold $H^{3} / \Gamma$. In fact $\Gamma$ can be taken as a subgroup of index twelve in the Picard group $P S L_{2} Z[i]$. As noted by Whitehead [1937], the complement $S^{3}-W$ is homeomorphic to the complement of the twisted version on the right. (Proof. Cut along a disk spanning $C$, rotate through any integral multiple of $2 \pi$, and then paste back together.) Now by filling in a solid torus neighborhood of $C$ we obtain the manifold $S^{3}-K$ from the hyperbolic manifold $S^{3}-(K \cup C) \cong S^{3}-W$. According to Thurston's theorem, this Dehn surgery must yield a new hyperbolic manifold, in all but a finite number of cases.

The knots $K$ obtained in this way are called Whitehead doubled knots. Thus Thurston shows that the complement of a Whitehead double has a hyperbolic structure whenever the number of twists is sufficiently large. Furthermore, the volumes of these manifolds tend from below to a finite limit. Note that the figure eight knot occurs as a special case.
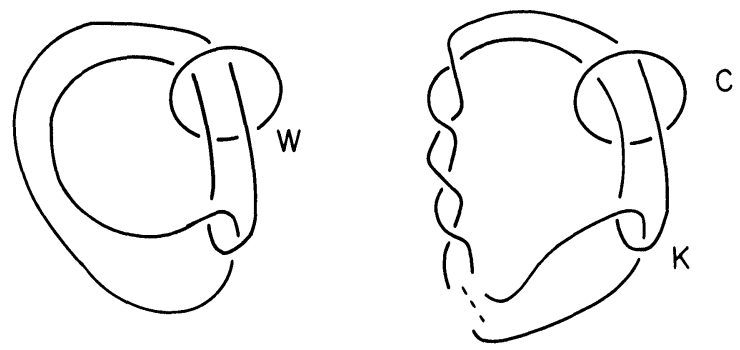

FIGURE 4. The Whitehead Link and a twisted version of it 
Appendix. Volume in hyperbolic 3-space. Lobachevsky's formulas for the volumes of certain polyhedra in hyperbolic space can best be expressed in terms of the function

$$
\pi(\theta)=-\int_{0}^{\theta} \log |2 \sin u| d u .
$$

The Russian $л$ is in honor of Lobachevsky, although he himself never used precisely this expression. Compare Clausen [1832], Coxeter [1935]. A graph is sketched in Figure 5. The basic properties can be listed as follows.

LEMMA 1. This function $\pi(\theta)$ is odd, periodic of period $\pi$, and satisfies the identity

$$
\pi(n \theta)=n \sum_{k \bmod n} \pi(\theta+k \pi / n)
$$

for every integer $n$.

Evidently, $л(\theta)$ is a continuous function, smooth except at the zeros of $\sin \theta$, taking its maximum at $\theta=\pi / 6$.

Proof of Lemma 1. Start with the trigonometric identity

$$
2 \sin n \theta=\prod_{k=0}^{n-1} 2 \sin (\theta+k \pi / n)
$$

which can be verified, for small positive values of $\theta$, by substituting $z=e^{-2 i \theta}$ in the equation

$$
\left|z^{n}-1\right|=\prod_{k \bmod n}\left|z-e^{2 \pi i k / n}\right| .
$$

The general case follows by analytic continuation.

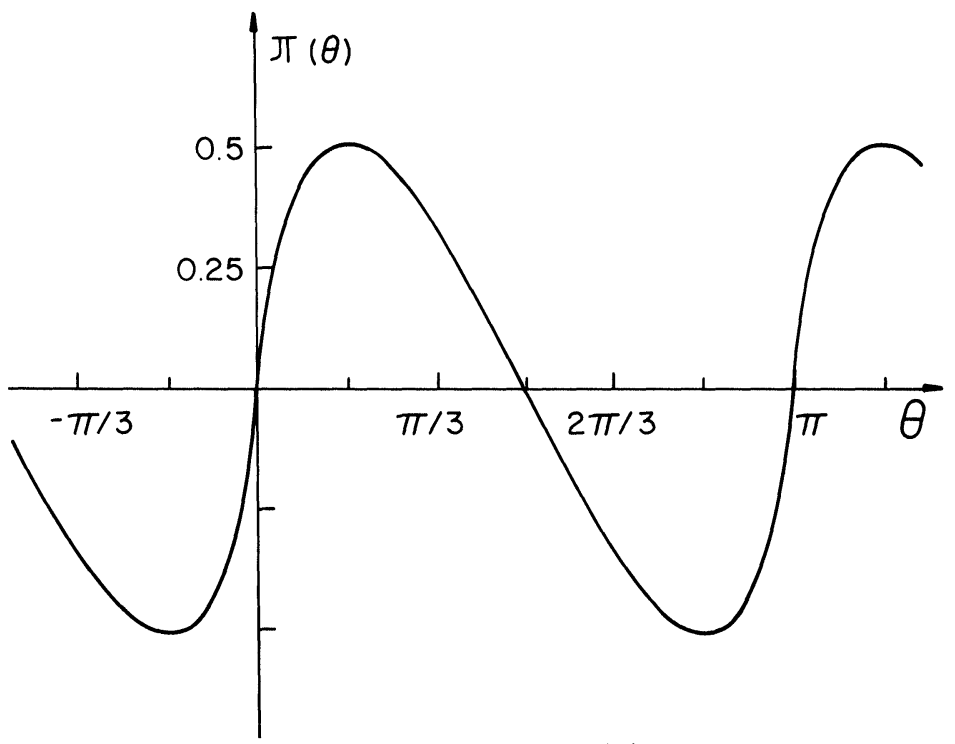

Figure 5. Graph of $\pi(\theta)$ 
Integrating the negative log absolute value of both sides we obtain

$$
\pi(n \theta) / n=\sum_{k=0}^{n-1} \pi(\theta+\pi k / n)+\text { constant }
$$

For example, if $n=2$, then

$$
\pi(2 \theta) / 2=\pi(\theta)+\pi(\theta+\pi / 2)+\text { constant. }
$$

Substituting $\theta=\pi / 2$ or $\theta=0$ in this last equation and subtracting, we see that $\pi(\pi)-л(0)=0$. Since the derivative $\pi^{\prime}(\theta)=-\log |2 \sin \theta|$ is periodic of period $\pi$, this proves that $\pi$ itself is also periodic of period $\pi$.

Finally, to prove that the constant in equation (1) is zero, we can simply average over all values of $\theta \bmod \pi$, noting that $\pi$ has average zero since it is an odd function.

This function $л(\theta)$ is closely related to the dilogarithm function

$$
L_{2}(z)=\sum z^{n} / n^{2}
$$

which has been studied by Euler, Abel, and many others. The precise relationship is described by the identity

$$
L_{2}\left(e^{2 i \theta}\right)=\pi^{2} / 6-\theta(\pi-\theta)+2 i \pi(\theta)
$$

for $0 \leqslant \theta \leqslant \pi$. (Compare Lewin [1958].) This identity can be verified by differentiating both sides for $0<\theta<\pi$, using the limiting value as $\theta \rightarrow 0$ to compute the constant $\pi^{2} / 6$.

Taking the imaginary part of both sides, we obtain the uniformly convergent Fourier series expansion

$$
\sum(\sin 2 n \theta) / n^{2}=2 \pi(\theta),
$$

which is valid for all values of $\boldsymbol{\theta}$.

For actual computation, it is much better to work with the series

$$
\pi(\theta)=\theta\left(1-\log |2 \theta|+\sum \frac{B_{n}(2 \theta)^{2 n}}{2 n(2 n+1) !}\right),
$$

which converges for $|\theta| \leqslant \pi$ and hence converges quite rapidly for small $\theta$. Here

$$
B_{1}=\frac{1}{6}, \quad B_{2}=\frac{1}{30}, \ldots
$$

are Bernoulli numbers. This equation can be proved by twice integrating the usual Laurent series expansion for $-\cot \theta$.

Here is an illustration of the utility of this function in computing hyperbolic volumes.

LEMMA 2. Consider an ideal hyperbolic 3-simplex, that is a simplex $\Delta$ with all four vertices on the sphere of points at infinity. If $\alpha, \beta, \gamma$ are the dihedral angles along three edges meeting at a common vertex, then $\alpha+\beta+\gamma=\pi$, and

$$
\operatorname{volume}(\Delta)=\pi(\alpha)+\pi(\beta)+\pi(\gamma) \text {. }
$$


It does not matter which particular vertex we choose, since it follows easily that opposite dihedral angles are equal so that we obtain the same three dihedral angles $\alpha, \beta, \gamma$ incident to any vertex.

This computation would follow easily from formulas of Lobachevsky [1836], although as far as I know he never worked out this particular example. (Compare Coxeter [1935].)

Proof. We will use the Beltrami upper half-space model, with metric $d s^{2}=\left(d x^{2}+d y^{2}+d z^{2}\right) / z^{2}$ and associated volume element $d x d y d z / z^{3}$, where $z>0$. Let us position the ideal simplex $\Delta$ so that one of its faces lies in the hemisphere $z=\left(1-x^{2}-y^{2}\right)^{1 / 2}$, and its opposite vertex lies at the infinite point. Projecting $\Delta$ orthogonally to the unit disk in the $x, y$-plane, we obtain a triangle inscribed in this disk with angles $\alpha, \beta, \gamma$. These are the angles of a euclidean triangle; so evidently $\alpha+\beta+\gamma=\pi$.

It follows easily from this picture that these angles determine $\Delta$ up to congruence (compare Andreev [1970]), and are subject to no other restrictions.

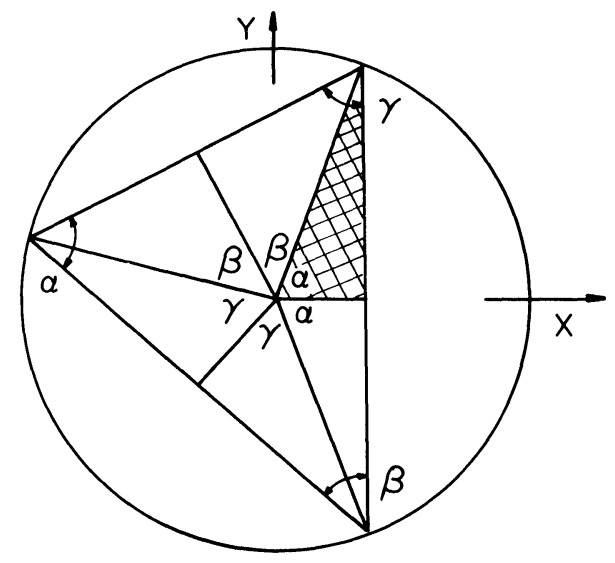

FIGURE 6. A triangle inscribed in the unit disk

If all three angles are acute, then barycentrically subdividing from the origin, we obtain six right triangles as illustrated in Figure 6. (The case of an obtuse angle can be handled by a similar argument, or by analytic continuation.) Take the region in the upper half space lying over just one of these triangles, namely the shaded one. We must integrate the Beltrami volume element $d x d y d z / z^{3}$ over the region defined by inequalities

$$
z \geqslant \sqrt{1-x^{2}-y^{2}}, \quad 0 \leqslant y \leqslant x \tan \alpha, \quad 0 \leqslant x \leqslant \cos \alpha .
$$

Integrating with respect to $z$, we obtain $\frac{1}{2} d x d y /\left(1-x^{2}-y^{2}\right)$. Integrating with respect to $y$, we obtain

$$
\frac{d x}{4 A}[\log (A+y)-\log (A-y)]_{0}^{x \tan \alpha}=\frac{d x}{4 A} \log \frac{A \cos \alpha+x \sin \alpha}{A \cos \alpha-x \sin \alpha}
$$


where $A=\left(1-x^{2}\right)^{1 / 2}$. Substituting $A=\sin \theta, x=\cos \theta, d x=-A d \theta$, and integrating, we get

$$
\begin{aligned}
\text { volume } & =\frac{1}{4} \int_{\alpha}^{\pi / 2} \log \frac{2 \sin (\theta+\alpha)}{2 \sin (\theta-\alpha)} d \theta \\
& =\left(-\pi\left(\frac{\pi}{2}+\alpha\right)+\pi(2 \alpha)+\pi\left(\frac{\pi}{2}-\alpha\right)-\pi(0)\right) / 4
\end{aligned}
$$

Now, if we substitute the identity

$$
\pi(2 \alpha)=2 \pi(\alpha)+2 \pi(\alpha+\pi / 2)
$$

this reduces easily to volume $=\pi(\alpha) / 2$. Adding the volumes of the other five regions, we obtain the required formula.

COROLlaRy. The maximum possible volume of a hyperbolic 3-simplex is $3 \pi(\pi / 3)$.

This works out as $1.0149416 \ldots$

Proof. We must maximize the continuous function $\pi(\alpha)+\pi(\beta)+\pi(\gamma)$ subject to the constraints $\alpha, \beta, \gamma \geqslant 0$ and $\alpha+\beta+\gamma=\pi$. Since the degenerate cases, where one dihedral angle is zero, all have zero volume, the maximum must occur at an interior point, where the derivative $\pi^{\prime}(\theta)=$ $-\log (2 \sin \theta)$ is defined and satisfies

$$
\pi^{\prime}(\alpha)=\pi^{\prime}(\beta)=\pi^{\prime}(\gamma)
$$

hence $\sin \alpha=\sin \beta=\sin \gamma$. Inspection shows that $\alpha=\beta=\gamma=\pi / 3$ is the only interior solution.

REMARK. Haagerup and Monkholm [1979] have proved the analogous theorem in $n$-dimensional hyperbolic space. The unique $n$-simplex of maximal volume is the regular ideal $n$-simplex.

Now let us compare Lemma 2 with Humbert's formulas for the volumes of certain fundamental domains. The presentation will be based on Borel [1981], which contains much further information about arithmetic computations of volume.

Let $\mathbf{Z}[\alpha]$ be the ring of integers in an imaginary quadratic number field $F=\mathrm{Q}[\alpha]$, with discriminant $-D=(\bar{\alpha}-\alpha)^{2}<0$; and let

$$
\Gamma=P S L_{2} \mathbf{Z}[\alpha] \subset P S L_{2} \mathbf{C}
$$

be the corresponding Bianchi group of isometries of hyperbolic 3-space. Borel shows that a fundamental domain for $\Gamma$ has

$$
\text { volume }=D^{3 / 2} \zeta_{F}(2) / 4 \pi^{2}
$$

where $\zeta_{F}$ is the Dedekind zeta function. Using the standard identity

$$
\zeta_{F}(s)=\zeta_{\mathbf{Q}}(s) \sum_{n=1}^{\infty}\left(\frac{-D}{n}\right) \frac{1}{n^{s}}
$$

(see for example Hecke [1923, §49]), where $\zeta_{Q}(2)=\pi^{2} / 6$, we obtain the formula

$$
\text { volume }=\frac{D^{3 / 2}}{24} \sum\left(\frac{-D}{n}\right) \frac{1}{n^{2}}
$$


of Humbert. Here $(-D / n)$ is a generalized quadratic residue symbol, with values \pm 1 or 0 . In order to express this infinite series in terms of the function $\pi(\theta)$, note first that the real-valued function $n \mapsto(-D / n)$ on $Z / D$ is equal to its own Fourier transform, up to a factor of $\sqrt{-D}=i \sqrt{D}$. More precisely

$$
\sum_{k \bmod D}\left(\frac{-D}{k}\right) e^{2 \pi i k n / D}=\left(\frac{-D}{n}\right) \sqrt{-D}
$$

for every positive integer $n$. (See Hecke $\S \S 52,58$.) Substituting this formula in (3), we obtain

$$
\text { volume }=\frac{D}{24 i} \sum_{k \bmod D}\left(\frac{-D}{k}\right) \sum_{n>1} e^{2 \pi i k n / D} / n^{2} .
$$

Evidently the real parts of the exponentials must cancel out. Hence, using the Fourier series expansion (2) we obtain

$$
\text { volume }=\frac{D}{12} \sum_{k \bmod D}\left(\frac{-D}{k}\right) \pi(k \pi / D) .
$$

Thus the volume of Humbert's fundamental domain is a finite linear combination, with rational coefficients, of values of the function $\pi(\theta)$ at rational multiples of $\pi$.

This suggests the conjecture that there may be some direct geometrical argument, describing such fundamental domains in terms of cutting and pasting of ideal simplices. (Compare Ash [1977].)

As an example, taking $D=3$ we obtain

$$
\text { volume }=\frac{1}{4}\left(\pi\left(\frac{\pi}{3}\right)-\pi\left(\frac{2 \pi}{3}\right)\right)=\frac{1}{2} \pi\left(\frac{\pi}{3}\right) .
$$

According to Riley, the figure eight knot complement must have volume equal to twelve times this number, or $6 \pi(\pi / 3)$. This is precisely twice the volume $3 л(\pi / 3)$ of a maximal hyperbolic 3-simplex, thus checking Thurston's observation that the figure eight complement can be triangulated by two copies of a maximal 3-simplex.

Such results suggest the interest of determining number theoretical properties of the real numbers $л(\theta)$. Here is an explicit guess.

CONJECTURE. If we consider only angles $\theta$ which are rational multiples of $\pi$, then every Q-linear relation

$$
q_{1} \pi\left(\theta_{1}\right)+\cdots+q_{n} \pi\left(\theta_{n}\right)=0
$$

is a consequence of the relations

$$
\begin{aligned}
\pi(\pi+\theta) & =\pi(\theta), \quad \pi(-\theta)=-\pi(\theta), \\
\pi(n \theta) & =n \sum_{k \bmod n} \pi(\theta+k \pi / n)
\end{aligned}
$$

of Lemma 1. 
For example $л(\pi / 3)$ and $\pi(\pi / 6)$ are subject to the Q-linear relation

$$
2 л(\pi / 6)=3 \pi(\pi / 3),
$$

as one easily verifies. But the conjecture would imply that $\pi(\pi / 3)$ and $\pi(\pi / 4)$ are rationally independent. In other words, no finite covering manifold of the figure eight complement should have the same volume as some covering of the Whitehead link complement.

A completely equivalent conjecture would be the following. For any fixed $n>2$, the $\varphi(n) / 2$ real numbers $\pi(k \pi / n)$, with $0<k<n / 2$ and $k$ relatively prime to $n$, are linearly independent over $\mathbf{Q}$. Here $\varphi$ is the Euler $\varphi$-function.

The author hopes to discuss this question further in a later paper. ${ }^{2}$

\section{REFERENCES}

[1827] C. F. Gauss, Disquisitiones generales circa superficies curvas, Commentationes Gottingensis 6 (Werke 4, 218-258; German summary, 341-347).

[1829-30] N. I. Lobachevsky, On the foundations of geometry, Kazan Messenger 25-28; German transl., 'Zwei geometrische Abhandlungen', Leipzig, 1898.

[1832] T. Clausen, Ueber die Function $\sin \varphi+\left(1 / 2^{2}\right) \sin 2 \varphi+\left(1 / 3^{2}\right) \sin 3 \varphi+$ etc., J. Reine Angew. Math. 8, 298-300.

J. Bolyai, The absolute science of space, independent of the truth or falsity of Euclid's Axiom 11 (which can never be proved a priori), Maros-Vásárhelyini.

[1836] N. I. Lobachevsky, Imaginary geometry and its application to integration, Kazan (German transl. Abh. Gesch. Math. 19, 1904).

[1837] __ Géometrie imaginaire, J. Reine Angew. Math. 17, 295-320.

[1859] A. Cayley, Sixth memoir upon quantics, Phil. Trans. 149, 61-91.

[1868] B. Riemann, Ueber die Hypothesen welche der Geometrie zu Grunde liegen, Abh. K. G. Wiss. Göttingen 13 (from his Inaugural Address of 1854).

E. Beltrami, Saggio di interpetrazione della geometria non-euclidea, Gior. Mat. 6, 248-312 (Also Op. Mat. 1, 374-405; Ann. École Norm. Sup. 6 (1869), 251-288).

, Teoria fondamentale degli spazii di curvatura costante, Annali di mat. ser. II 2 , 232-255 (Op. Mat. 1, 406-429; Ann. École Norm. Sup. 6 (1869), 345-375).

[1871] F. Klein, Uber die sogenannte Nicht-Euklidische Geometrie, Math. Ann. 4, 573-625 (cf. Ges. Math. Abh. 1, 244-350).

E. Betti, Sopra gli spazi di un numero qualunque di dimensioni, Annali di mat. ser. II 4, 140-158.

[1873] H. A. Schwarz, Ueber diejenigen Fälle in welchen die Gaussische hypergeometrische Reihe eine algebraische Function ihres vierten Elementes darstellt, J. Reine Angew. Math. 75, 292-335 (Ges. Math. Abh. 2, 211-259).

[1882] H. Poincaré, Theorie des groupes fuchsiens, Acta Math. 1, 1-62; (Oeuv. 2, 108-168).

[1883] __ Mémoire sur les groupes kleinéens, Acta Math. 3, 49-92; (Oeuv. 2, 258-299).

[1884] E. Picard, Sur un groupe de transformations des points de l'espace situés du même côté d'un plan, Bull. Soc. Math. France 12, 43-47.

[1890] W. Dyck, Beiträge zur Analysis situs. II, Math. Ann. 37 273-316.

F. Klein, Zur Nicht-Euklidischen Geometrie, Math. Ann. 37, 544-572 (Ges. Abh. 1, 353-383).

[1891] L. Bianchi, Geometrische Darstellung der Gruppen Linearer Substitutionen mit ganzen complexen Coefficienten nebst Anwendungen auf die Zahlentheorie, Math. Ann. 38, 313-333.

\footnotetext{
2“On polylogarithms, Hurwitz zeta functions and the Kubert identities” (in preparation).
} 
W. Killing, Ueber die Clifford-Klein'schen Raumformen, Math. Ann. 39, 258-278.

[1895] H. Poincaré, Analysis situs, J. École Polyt. 1, 1-121 (Oeuv. 6, 193-288).

[1898] J. Hadamard, Les surfaces à courbures opposées et leur lignes géodésiques, J. Math. Pures Appl. Ser. 5 4, 27-73 (Oeuv. 2, 729-775).

[1900] C. F. Gauss, .Werke 8, 175-239.

[1908] H. Tietze, Uber die topologischen Invarianten mehrdimensionaler Mannigfaltigkeiten, Monatshefte für Math. Phys. 19, 1-118.

[1912] H. Gieseking, Analytische Untersuchungen ueber topologische Gruppen, Thesis, Muenster (Compare Magnus (1974), 153).

L. E. J. Brouwer, Über Abbildung von Mannigfaltigkeiten, Math. Ann. 71, 97-115.

[1913] O. Veblen and J. W. Alexander, Manifolds of $n$ dimensions, Ann. of Math. (2) 14, 163-178.

H. Weyl, Die Idee der riemannschen Fläche, Teubner, Leipzig.

[1914] F. Hausdorff, Grundzüge der Mengenlehre, von Veit, Leipzig.

[1919] G. Humbert, Sur la mesure des Classes d'Hermite de discriminant donné dans un corps quadratique imaginaire, et sur certains volumes non euclidiens, Comptes Rendus (Paris) 169, 448-454.

[1923] E. Hecke, Vorlesungen über die Theorie der algebraischen Zahlen, Akad. Verl., Leipzig.

[1925] H. Hopf, Zum Clifford-Kleinschen Raumproblem, Math. Ann. 95, 313-339.

[1928] E. Cartan, Leçons sur la géométrie des espaces de Riemann, Gauthier-Villars, Paris.

[1931] H. Hopf and W. Rinow, Über den Begriff der vollständigen differentialgeometrischen Fläche, Comment. Math. Helv. 3, 209-225.

[1935] H. S. M. Coxeter, The functions of Schläfli and Lobatschefsky, Quart. J. Math. 6, 13-29 (pp. 3-20 of twelve geometric essays, S. Ill. Univ. Press, Carbondale, 1968).

[1937] J. H. C. Whitehead, On doubled knots, J. London Math. Soc. 12, 63-71 (Works 2, 59-67).

[1942] H. S. M. Coxeter, Non-Euclidean geometry, Univ. Toronto Press, Toronto.

[1958] L. Lewin, Dilogarithms and associated functions, MacDonald, London.

[1963] A. Borel, Compact Clifford-Klein forms of symmetric spaces, Topology 2, 111-112.

[1970] G. A. Margulis, Isometry of closed manifolds of constant negative curvature with the same fundamental group, Soviet Math. Dokl. 11, 722-723.

E. M. Andreev, On convex polyhedra [of finite volume] in Lobačevskil space, Mat. Sbornik 81, 445-478 and 83, 256-260 (transl. 10, 413-440 and 12, 255-259).

[1971] G. D. Mostow, The rigidity of locally symmetric spaces, Proc. (1970) Internat. Congr. Math., vol. 2, pp. 187-197, Gauthier-Villars, Paris.

[1973] _ Strong rigidity of locally symmetric spaces, Ann. Math. Studies, No. 78, Princeton Univ. Press, Princeton, N. J.

G. Prasad, Strong rigidity of Q-rank 1 lattices, Invent. Math. 21, 255-286.

[1974] W. Magnus, Noneuclidean tesselations and their groups, Academic Press, New York.

[1975] R. Riley, A quadratic parabolic group, Math. Proc. Cambridge Philos. Soc. 77, 281-288.

[1977] A. Ash, Deformation retracts with lowest possible dimension of arithmetic quotients of self-adjoint homogeneous cones, Math. Ann. 225, 69-76.

[1978] W. Thurston, Geometry and 3-manifolds, Lecture Notes, Princeton University. N. Wielenberg, The structure of certain subgroups of the Picard group, Math. Proc. Cambridge Philos. Soc. 84, 427-436.

[1979] U. Haagerup and H. Munkholm, Simplices of maximal volume in hyperbolic n-space, preprint, Odense University.

[1980] H. J. Munkholm, Simplices of maximal volume in hyperbolic space, Gromov's norm, and Gromov's proof of Mostow's rigidity theory (following Thurston), pp. 109-124 of Topology Symposium-Siegen 1979, ed. Koschorke and Neumann, Springer Lecture Notes in Math., no. 788.

R. Rieley, Applications of a computer implementation of Poincarés theorem on fundamental polyhedra, preprint, I.A.S.

, Seven excellent knots, preprint, I.A.S.

M.-F. Vignéras, Variétés riemanniennes isospectrales et non isométriques, Ann. of Math. (2) 112, 21-32. 
[1981] A. Borel, Commensurability classes and volumes of hyperbolic 3-manifolds, Ann. Scuola Norm. Sup. Pisa Cl. Sci. (4) 8, 1-33.

M. Gromov, Hyperbolic manifolds according to Thurston and Jorgensen, Sémin. Bourbaki 546, Springer Lecture Notes in Math., no. 842, pp. 40-53.

D. Sullivan, Travaux de Thurston sur les groupes quasi-fuchsiens et les variétés hyperboliques de dimension 3 fibrées sur $S^{1}$, Sémin. Bourbaki 554, Springer Lecture Notes in Math., no. 842, pp. 196-214.

W. Thurston, Three dimensional manifolds, Kleinian groups and hyperbolic geometry (to appear). (to appear).

Hyperbolic structures on 3-manifolds, I: Deformation of acylindrical manifolds

School of Mathematics, Institute for Advanced Study, Princeton, New Jersey 08540 